\title{
Mechanical Quadrature Near a Singularity
}

The purpose of this note is to present coefficients to facilitate computation of integrals of the type $\int_{0}^{b} x^{-\frac{1}{3}} f(x) d x$. If the integrand $x^{-\frac{1}{3}} f(x)$ has no singularity at the origin, the coefficients presented are still applicable, though in this case other well-known procedures for numerical integration might be more appropriate. In this regard, it should be noted that suitable modifications of $f(x)$ permit one to employ the coefficients in evaluating $\int_{0}^{b} x^{\frac{1}{3}} f(x) d x$. In applied problems, the analytical form of $f(x)$ is usually known though it may be rather complicated in form. The singularity may be removed by imposing the transformation $x=y^{2}$ or by fractional integration after which any one of a number of numerical procedures can be applied. The former approach is not satisfactory since the upper limit of integration as a rule becomes irrational and so complicates tabulation of the resulting integrand. Integration by parts requires the computation of $f^{\prime}(x)$. If $f(x)$ is quite complicated, its derivative is usually even more so, and again an excessive amount of tabulation is required. A third possibility is to expand $f(x)$ into a power series about the origin. However, in many cases this is not easy owing to the complexity of $f(x)$. Furthermore, the series may be slowly convergent. A more satisfactory procedure is to write the Lagrangian polynomial which precisely fits $f(x)$ at equally spaced points over the interval $(0, b)$. Then a numerical integration formula is easily evolved by calculating the moments $\int_{0}^{b} x^{-\frac{1}{1}} x^{m} d x, m=0,1,2, \cdots$. It is convenient to consider the integral in the form $A_{n}=\int_{0}^{n h} x^{-\frac{1}{2}} f(x) d x$. Let $f_{r}=f(r h), r=0,1, \cdots, n$ and write

$$
A_{n}=2(n h)^{\frac{1}{2}}\left(\sum_{r=0}^{n} f_{r} \gamma_{r}^{(n)}\right) D_{n}^{-1}+R_{n}
$$

where $R_{n}$ is the remainder term. Following the procedure described above, the exact coefficients $\gamma_{r}{ }^{(n)}$ and $D_{n}$ are given in Table I for $n=1(1) 10$.

It is clear that the $(n+1)$ point formula so derived is exact if $f(x)$ is a polynomial of the $n$th degree. If $f(x)$ is not of this form, it is of interest to examine the remainder. Evaluation of the remainder term in linear methods of approximation has been discussed by MiLNE. ${ }^{1,2}$ Following his notation, the remainder is given by

$$
R_{n}=\int_{0}^{n h} f^{(n+1)}(s) G(s) d s .
$$

The analytical form of $G(s)$ is easily deduced from the approximating formula. For the $(n+1)$ point formula

$$
n ! G(s)=\int_{s}^{n h} x^{-\frac{1}{2}}(x-s)^{n} d x-2(n h)^{\frac{1}{2}} \sum_{r=0}^{n} \overline{(r h-s)^{n}} \gamma_{r}{ }^{(n)} / D_{n},
$$


where

$$
\begin{aligned}
(\overline{r h-s})^{n} & =(r h-s)^{n} & \text { if } r h \geq s \\
& =0 & \text { if } r h<s .
\end{aligned}
$$

The formula for $A_{n}$ is exact if $f(x)=(x-s)^{n}$ and so

$$
0=\int_{0}^{n h} x^{-3}(x-s)^{n} d x-2(n h)^{1} \sum_{r=0}^{n}(r h-s)^{n} \gamma_{r}^{(n)} / D_{n} .
$$

Suppose $0 \leq s \leq h$. Subtracting (5) from (3) and using (4), a straightforward reduction gives

(6) $n ! G(s)=2(-1)^{n}(n h)^{\frac{1}{2}} \gamma_{0}^{(n)} s^{n}-\frac{2^{n+1}(-1)^{n} n ! s^{n+1}}{1 \cdot 3 \cdot 5 \cdots \cdots(2 n+1)}$ if $0 \leq s \leq h$.

Denote the right hand side of (5) by $G_{1}(s)$. Let

$$
\begin{aligned}
H_{r}(s) & =2(n h)^{\frac{1}{3}}(r h-s)^{n} \gamma_{r}^{(n)} / D_{n} ; & \\
G_{r}(s) & =G_{r-1}(s)+H_{r-1}(s), & r=2,3, \cdots, n .
\end{aligned}
$$

Then

$$
n ! G(s)=G_{r}(s) \text { if }(r-1) h \leq s \leq r h, \quad r=1,2, \cdots, n .
$$

We extend some terminology by Milne and say that $G(s)$ is definite if it does not change sign in the interval of $(0, n h)$; otherwise, it is indefinite. If $G(s)$ is definite, application of the mean value theorem to (2) gives

$$
R_{n}=f^{(n+1)}(\theta) \int_{0}^{n h} G(s) d s,
$$

where $0<\theta<n h$. In any event, bounds for the error are given by

$$
\begin{gathered}
\left|R_{n}\right|<M \int_{0}^{n h}|G(s)| d s \text { or }\left|R_{n}\right|<M K n h, \\
M=\operatorname{Max}_{0 \leq s \leq n h}\left|f^{(n+1)}(s)\right|, \quad K=\underset{0 \leq s \leq n h}{\operatorname{Max}}|G(s)| .
\end{gathered}
$$

If $G(s)$ is indefinite, it must vanish at least once in the open interval $(0, n h)$ (observe that $G(0)=G(n h)=0$ ). Suppose $\xi$ is the one and only one such point. Then the remainder may be composed of two parts.

$$
R_{n}=f^{(n+1)}\left(\theta_{1}\right) \int_{0}^{\xi} G(s) d s+f^{(n+1)}\left(\theta_{2}\right) \int_{\xi}^{n h} G(s) d s
$$

where $0<\theta_{1}<\xi<\theta_{2}<n h$. The extension of this argument for $G(s)$ vanishing at more than one point in the open interval of integration is obvious.

It is known that $\int_{0}^{n h} G(s) d s$ may be evaluated directly from the approximating formula without a knowledge of $G(s)$. In the present instance

$$
\int_{0}^{n h} G(s) d s=2(n h)^{\frac{t}{t}} h^{n+1}\left\{n^{n+1} /(2 n+3)-\sum_{r=1}^{n} r^{n+1} \gamma_{r}^{(n)} / D_{n}\right\} /(n+1) !
$$

Employing the remainder term for the polynomial approximation to $f(x)$, 
an expression equivalent to (12) is

$$
\int_{0}^{n h} G(s) d s=\int_{0}^{n h} x^{3}(x-h)(x-2 h) \cdots(x-n h) d x /(n+1) !
$$

Thus, if $G(s)$ is definite, computation of a rigorous error term is considerably simplified. As to the definiteness of $G(s)$, no general theorem is available. But employing (8), we can compute $G(s)$ for each formula and ascertain in a heuristic fashion if it is definite. In this study, $G(s)$ is definite for all the even point formulas. For the odd point formulas, $G(s)$ vanishes at one point in the open interval $(0, n h)$. For each integration formula given in Table I, the exact value of $\int_{0}^{n h} G(s) d s$ is presented in Table II. For the odd point formulas, the values of $\xi, \int_{0}^{\xi} G(s) d s$ and $\int_{\xi}^{n h} G(s) d s$ are also tabulated; the first mostly to $5 \mathrm{D}$, the latter to $6 \mathrm{D}$.

The coefficients $\gamma_{r}{ }^{(n)}$ were checked by verifying that (1) is exact for $f(x)=x^{m}, m=0,1, \cdots, n$. The values of $\int_{0}^{n h} G(s) d s$ were obtained employing (12) and checked by (13) and integration of (8). The function $G(s)$

TABLE I

VALUES OF $\boldsymbol{\gamma}_{\boldsymbol{r}}^{(n)}$ AND $D_{n}$

In each column headed by $n$, the first coefficient is $\gamma_{0}^{(n)}$, the second is $\gamma_{1}^{(n)}$, etc. The last number is the value of $D_{n}=\sum_{r=0}^{n} \gamma_{r}^{(n)}$.




TABLE II

ERRor CoEfFICIENTS

$\begin{array}{rcccc}n & \int_{0}^{n h} G(s) d s / h^{n+1}(n h)^{1 / 2} & \xi / h & \int_{0}^{\xi} G(s) d s / h^{n+3 / 2} & \int_{\xi}^{\mathrm{nh}} G(s) d s / h^{n+3 / 2} \\ 1 & -2 / 15 & & & \\ 2 & 8 / 315 & 1.24092 & 0.039468 & -0.003551 \\ 3 & -1 / 35 & & & \\ 4 & 16 / 1485 & 2.69858 & 0.022193 & -0.000644 \\ 5 & -1018 / 81081 & & & \\ 6 & 152 / 25025 & 4.11461 & 0.015041 & -0.000163 \\ 7 & -35098 / 4922775 & & & \\ 8 & 18095776 / 4583103525 & 5.53190 & 0.011218 & -0.000050 \\ 9 & -2620473 / 565815250 & & & -0.000017 \\ 10 & 106115816 / 37948097187 & 6.9556 & 0.008860 & \end{array}$

was expanded in a Taylor series about an approximate value of $\xi$, and $\xi$ was determined by inversion of this series. Taylor's formula was also used to calculate $\int_{0}^{\xi} G(s) d s$. The latter calculations were performed in duplicate and are correct to within one unit of the last decimal place given.

It appears that coefficients of the kind presented here were first derived in a thesis by M. BATES. ${ }^{3}$ This work gives exact coefficients for $\int_{0}^{n h} x^{m / 2} f(x) d x, m= \pm 1, n=2(1) 4$. The remainder terms are examined in some detail. In a thesis by M. E. YounGBERG, ${ }^{4}$ similar formulas were given for $n=3(1) 7$. With the exception of the seven- and eight-point formulas (the eight-point formula is totally in error), exact coefficients are also tabulated to enable integration over any sub-interval defined by the points at which $f(x)$ is tabulated. No estimates of the error are given.

In a recent paper, E. L. Kaplan ${ }^{b}$ has derived three- and five-point coefficients for the evaluation of $\int_{r_{h} h}^{r_{2} h} x^{m / 2} f(x) d x, m= \pm 1$. All coefficients are in decimal form. For three points, coefficients are given for $r_{1}=0(1) 18$. In addition, some coefficients are presented so that, using three ordinates, the integral over the first two or last two ordinates can be found. The fivepoint case is similarly treated, but the values permit integration over three ordinates at most. Two sets of coefficients are tabulated. One is exact for consecutive powers of $x$ as in our case. The other is exact for $f(x)$ an even function. Finally, error terms are given for selected cases having the former property. In effect, it is assumed that the mean value theorem is applicable and the error coefficients are derived by evaluating equations of the type as of the right hand side of (12). But the author rightly remarks that the results must be used with caution if $r_{2}-r_{1}>1$. As a further aid in evaluating the remainder, Kaplan also gives values which account for the second approximated term in the Taylor series expansion of $f(x)$. We have examined the $G(s)$ function for $n=2, r_{1}=4, r_{2}=6$ and $m=-1$ and find that it too vanishes in the open interval of integration. The same is true for the three-point formula where $r_{1}=0, r_{2}=2$ and $m=1$. 
The author acknowledges with thanks the aid of Dolores UFForD, who assisted in the calculations.

Midwest Research Institute

YUdell L. LuKE

Kansas City 2, Missouri

${ }^{1} \mathrm{~W}$. E. Milne, "The remainder in linear methods of approximation," NBS, $J n$. of Research, v. 43, 1949, p. 501-511.

2 W. E. Milne, Numerical Calculus. p. 108-116.

${ }^{3}$ M. BATES, On the Development of Some New Formulas for Numerical Integration. Stanford University, June, 1929.

${ }^{4}$ M. E. YoungBerg, Formulas for Mechanical Quadrature of Irrational Functions. Oregon State College, June, 1937. (The author is indebted to the referee for references 3 and 4.)

5E. L. Kaplan, "Numerical integration near a singularity," Jn. Math. Phys., v. 26, April, 1952, p. 1-28.

\section{On the Numerical Solution of Equations Involving Differential Operators with Constant Coefficients}

1. The General Linear Differential Operator. Consider the differential equation of order $n$

$$
L y+F(y, x)=0,
$$

where the operator $L$ is defined by

$$
L y=\sum_{k=0}^{n} P_{k}(x) \frac{d^{k} y}{d x^{k}},
$$

and the functions $P_{k}(x)$ and $F(y, x)$ are such that a solution $y$ and its first $n$ derivatives exist in $0 \leq x \leq X$. In the special case when (1) is linear the solution can be completely determined by the well known method of variation of parameters when $n$ independent solutions of the associated homogeneous equations are known. Thus for the case when $F(y, x)$ is independent of $y$, the solution of the non-homogeneous equation can be obtained by mere quadratures, rather than by laborious stepwise integrations. It does not seem to have been observed, however, that even when $F(y, x)$ involves the dependent variable $y$, the numerical integrations can be so arranged that the contributions to the integral from the upper limit at each step of the integration, at the time when $y$ is still unknown at the upper limit, drop out. Thus again the computation can be made to involve merely quadratures.

It is not of ten that the solution of the homogeneous equation can be simply determined, and it is perhaps for this reason that attention has not been given heretofore to the possibility of simplifying the numerical evaluation of the solution by making use of the solutions to the homogeneous equation. However, in the case when the functions $P_{k}(x)$ in $L$ are constants, the solution of the homogeneous equation is easy to determine. This is particularly true when the order of the differential equation is fairly low. In the instance when the operator $L$ is of second order, with constant coefficients, the method of using the integral equation often has decided advantages over the usual methods employed for solving differential equations. 